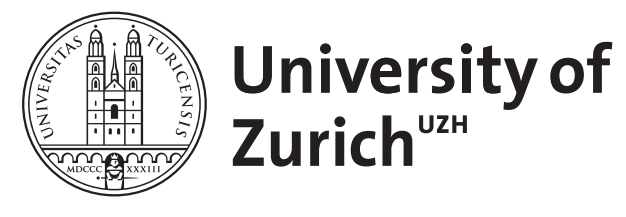

\title{
Noise reinforcement for Lévy processes
}

\author{
Bertoin, Jean
}

\begin{abstract}
In a step reinforced random walk, at each integer time and with a fixed probability p (0, 1 ), the walker repeats one of his previous steps chosen uniformly at random, and with complementary probability 1 - p, the walker makes an independent new step with a given distribution. Examples in the literature include the so-called elephant random walk and the shark random swim. We consider here a continuous time analog, when the random walk is replaced by a Lévy process. For sub-critical (or admissible) memory parameters $\mathrm{p}<\mathrm{pc}$, where pc is related to the Blumenthal-Getoor index of the Lévy process, we construct a noise reinforced Lévy process. Our main result shows that the step-reinforced random walks corresponding to discrete time skeletons of the Lévy process, converge weakly to the noise reinforced Lévy process as the time-mesh goes to 0 .
\end{abstract}

DOI: https://doi.org/10.1214/19-aihp1037

Posted at the Zurich Open Repository and Archive, University of Zurich

ZORA URL: https://doi.org/10.5167/uzh-190454

Journal Article

Published Version

Originally published at:

Bertoin, Jean (2020). Noise reinforcement for Lévy processes. Annales de l'Institut Henri Poincaré (B) Probabilities et Statistiques, 56(3):2236-2252.

DOI: https://doi.org/10.1214/19-aihp1037 


\title{
Noise reinforcement for Lévy processes
}

\author{
Jean Bertoin \\ Institute of Mathematics, University of Zurich, Zurich, Switzerland. E-mail: jean.bertoin@math.uzh.ch
}

Received 17 October 2018; revised 4 September 2019; accepted 1 November 2019

\begin{abstract}
In a step reinforced random walk, at each integer time and with a fixed probability $p \in(0,1)$, the walker repeats one of his previous steps chosen uniformly at random, and with complementary probability $1-p$, the walker makes an independent new step with a given distribution. Examples in the literature include the so-called elephant random walk and the shark random swim. We consider here a continuous time analog, when the random walk is replaced by a Lévy process. For sub-critical (or admissible) memory parameters $p<p_{c}$, where $p_{c}$ is related to the Blumenthal-Getoor index of the Lévy process, we construct a noise reinforced Lévy process. Our main result shows that the step-reinforced random walks corresponding to discrete time skeletons of the Lévy process, converge weakly to the noise reinforced Lévy process as the time-mesh goes to 0 .
\end{abstract}

Résumé. Dans une marche aléatoire à pas renforcés, à chaque instant entier et avec une probabilité fixée $p \in(0,1)$, le marcheur répète un de ses précédents pas tiré uniformément au hasard, et avec probabilité $1-p$ effectue un nouveau pas indépendant de loi donnée. Comme exemples dans la littérature figurent l'elephant random walk et le shark random swim. Nous nous intéressons ici à un analogue en temps continu, c'est-à-dire lorsque la marche aléatoire est remplacée par un processus de Lévy. Pour des paramètres de mémoire sous-critiques (ou encore admissibles) $p<p_{c}$, où $p_{c}$ est lié à l'indice de Blumenthal-Getoor du processus de Lévy, nous construisons un processus de Lévy à bruit renforcé. Notre résultat principal établit la convergence en loi des marches aléatoires à pas renforcés associées aux squelettes discrets d'un processus de Lévy, vers le processus de Lévy à bruit renforcé, lorsque que le pas de la subdivision du temps tend vers 0 .

MSC: 60G50; 60G51; 60K35

Keywords: Reinforcement; Preferential attachment; Lévy process; Yule-Simon distribution; Blumenthal-Getoor index

\section{Introduction}

The terminology random walk is commonly used in the literature for two different families of stochastic processes in discrete time. First, a random walk can refer to a Markov chain on a graph, such that one-step transitions are allowed only between neighboring vertices. Second, a random walk can also refer to the sequence of the partial sums of i.i.d. random variables, say in $\mathbb{R}^{d}$. Although the main part of this work is concerned with the second meaning only, we shall need in this introduction both interpretations, and it should be clear from the context which one is actually in use.

Informally, the edge reinforced random walk, which has been introduced in 1986 by Coppersmith and Diaconis in a frequently cited unpublished article, is non-Markovian stochastic process on a locally finite graph, which tends to travel more often through edges it has already often travelled through in the past. More precisely, the conditional probability that the $n$th step of the process is from a vertex $x$ to a neighboring vertex $y$ given the trajectory up to time $n$, is proportional to $a_{e}$ plus the number of times the edge $e=\{x, y\}$ has been previously crossed, where $a_{e}>0$ represents the initial weight of the edge $e$. This model and its variations (reinforcement can be non-linear, or rather concern vertices instead of edges, ...) have triggered numerous works over the last decades. Pemantle [17] wrote a most useful survey some 12 years ago; we also refer to $[1,9,10,15,18]$ and works cited therein for some more recent developments.

The motivation for the present paper stems from a related notion of reinforcement, now for the second meaning of random walk. Informally, a step reinforced random walk tends to repeat steps that it has already often made in the past. Specifically, consider a random walk $S(n)=X_{1}+\cdots+X_{n}$ where the steps $X_{1}, X_{2}, \ldots$ form a sequence of i.i.d. variables in $\mathbb{R}^{d}$. We update the increments $X_{i}$ one after another as follows. Fix some $p \in(0,1)$, called the memory parameter, and let $\left(\varepsilon_{i}: i \geq 2\right)$ be an independent sequence of Bernoulli variables with parameter $p$. We set first $\hat{X}_{1}=X_{1}$, and next for 
$i \geq 2$, we let $\hat{X}_{i}=X_{i}$ if $\varepsilon_{i}=0$, whereas if $\varepsilon_{i}=1$, then we define $\hat{X}_{i}$ as a uniform random sample from $\hat{X}_{1}, \ldots, \hat{X}_{i-1}$. Finally, the sequence of the partial sums of the updated sequence,

$$
\hat{S}(n)=\hat{X}_{1}+\cdots+\hat{X}_{n}, \quad n \in \mathbb{N},
$$

is called a step reinforced random walk. In words, at each time, with probability given by the memory parameter $p, \hat{S}$ repeats one of its preceding steps chosen uniformly at random, and otherwise $\hat{S}$ has an independent increment with a fixed distribution. In the memoryless case $p=0$, one has $\hat{S}(n)=S(n)$, whereas $\hat{S}(n)=n X_{1}$ in the perfect memory case $p=1$. So, say when the steps are centered with finite variance, one naturally expects that the asymptotic behavior of the step reinforced random walk with memory $p \in(0,1)$ should somehow interpolate between the diffusive behavior and the ballistic behavior with a random velocity.

Step reinforcement has been considered for the one-dimensional simple symmetric random walk, i.e. $d=1$ and $\mathbb{P}\left(X_{i}=\right.$ 1) $=\mathbb{P}\left(X_{i}=-1\right)=1 / 2$, by Kürsten [14] as a version of the so-called elephant random walk. ${ }^{1}$ The latter is a model of a random walk with memory which had been introduced previously by Schütz and Trimper [19] and then studied by several authors, see e.g. [2,3,7,8,14]. Quite recently, Businger [5] investigated the scaling limits of the so-called shark random swim, a step reinforced random walk whose increments follow an isotropic stable distribution in $\mathbb{R}^{d}$. A striking feature that has been pointed at in those works is that the long-time behavior of $\hat{S}$ exhibits a phase transition at some critical memory parameter. Specifically, in the simple symmetric case, $n^{-1 / 2} \hat{S}(n)$ converges in distribution to some Gaussian law when $p<1 / 2$, whereas for $p>1 / 2, n^{-p} \hat{S}(n)$ converges almost surely to some (non Gaussian) random variable. If the steps follow an isotropic stable law with index $\alpha \in(0,2]$, then $n^{-1 / \alpha} \hat{S}(n)$ converges in distribution to some stable law when $\alpha p<1$, whereas $n^{-p} \hat{S}(n)$ converges almost surely to some (non zero) random variable when $\alpha p>1$.

The purpose of the present work is to investigate the analog of step reinforcement in continuous time, that when the random walk $S=(S(n))_{n \in \mathbb{N}}$ is replaced by a Lévy process $\xi=(\xi(t))_{t \geq 0}$, for memory parameters smaller than a certain critical value. Informally, the steps $X_{i}$ of $S$ need to be substituted by the increments of $\xi$ on infinitesimal time durations, that is by a Lévy noise $\mathrm{d} \xi(t)$. We shall argue that the existence of a stochastic process $\hat{\xi}$ whose time-derivative $\mathrm{d} \hat{\xi}(t)$ in the sense of generalized functions can be viewed as a reinforcement of the Lévy noise $\mathrm{d} \xi(t)$, depends crucially on the so-called Blumenthal-Getoor index $\beta$ of $\xi$. The latter was introduced in [4], and it is well-known that several local path properties of $\xi$ are then similar to those of a $\beta$-stable Lévy process. We refer to the introduction in [16] for some background and historical perspective on this notion. We shall see that $p_{c}=1 / \beta$ is the critical memory parameter for noise reinforcement of Lévy processes, specifically $\hat{\xi}$ can be defined when the memory parameter $p$ is admissible, that is smaller than $1 / \beta$, but this is no longer possible when $p \beta>1$. The most interesting situation is when $\beta>1$, as otherwise the inequality $p \beta<1$ always holds. The Lévy process $\xi$ then has a.s. bounded $\gamma$-variation for $\gamma>\beta$, and a.s. infinite $\gamma$-variation for $\gamma<\beta$. Informally, reinforcing the Lévy noise $\mathrm{d} \xi(t)$ with a super-critical memory parameter $p>1 / \beta$ disrupts the compensation mechanism at work for Lévy processes with unbounded variation to the point that the reinforced noise cannot be integrated.

An important general question about reinforced processes is to describe in which ways reinforcement alters the asymptotic behavior (recurrence, transience, trapping, asymptotic velocity, etc.; see e.g. $[1,9,10,17,18]$ ). Because of the repetition of certain jumps, a noise reinforced Lévy process is usually not Markovian, and its distribution is singular with respect to that of the original Lévy process. It is thus natural to ask similarly how noise reinforcement affects the local properties of the Lévy process (oscillating behavior, rate of growth, etc.); however this will not be tackled here.

The rest of this work is organized in two main parts: in the first, we construct noise reinforced Lévy processes, and in the second, we consider random walks arising as discrete time skeletons of a Lévy process and establish the weak convergence of the step reinforced random walks to the noise reinforced Lévy process as the time-mesh goes to 0 . More precisely, the plan is as follows.

Section 2.1 introduces Yule-Simon processes, a family of counting processes with one-dimensional marginal laws closely related to so-called Yule-Simon distributions, and which form the building blocks for noise reinforcement. The material is elementary and doubtless already well-known, although I have often been unable to find precise references for some of the basic results which are discussed there. In Section 2.2, we investigate convergence of random sums of independent Yule-Simon processes, possibly after an adequate compensation, and point at the key role of the BlumenthalGetoor index in this framework. This is used in Section 2.3 to construct noise reinforced Lévy processes, essentially by mimicking the celebrated Lévy-Itô decomposition; an important feature is that one can compute the multidimensional characteristic functions of such processes in terms of certain functionals of Yule-Simon processes. Our main result is claimed and established in Section 3: when the memory parameter $p$ is admissible, then the step reinforced random walk

\footnotetext{
${ }^{1}$ Beware that most works on elephant random walks use a different - but actually equivalent - construction, where the memory parameter corresponds to $(p+1) / 2$ in the present notation.
} 
associated to a discrete time skeleton of a Lévy process $\xi$ converges in distribution to the corresponding noise reinforced Lévy process $\hat{\xi}$ as the time-mesh tends to 0 . The proof relies on the convergence of the empirical measure of the counting processes for occurrences in Simon's reinforcement model [20] towards the law of a Yule-Simon process, which requires techniques of propagation of chaos, and a uniform-integrability property of those counting processes. The fact that a phase transition occurs at the critical memory parameter $p_{c}=1 / \beta$ is essentially a consequence of the property that Yule-Simon distributions are heavy-tailed.

\section{Construction of noise-reinforced Lévy processes}

\subsection{Yule-Simon processes}

Simon [20] introduced a simple random dynamic for explaining the appearance of a family of heavy-tailed distributions in a variety of empirical data in biology, sociology, economics, ... It can be viewed as an early prototype of preferential attachment models later popularized by Barabási and Albert, to which the step reinforced random walk is closely related. Typically, imagine one writes a book as follows, producing a large random sequence of words. Fix a parameter $p \in(0,1)$, and write the first word of the book. Next, repeat this same word with probability $p$, or write a new word with probability $1-p$. When the length of the text is $k \geq 2$, the event that the $(k+1)$ th word of the text is a new one which has not appeared previously has probability $1-p$, and on the complementary event, the $(k+1)$ th word of the text is sampled uniformly at random among its $k$ first words. The book is completed when the text has reached a large length (i.e. number of words) $n \gg 1$; one is then interested in the statistics of occurrences of words, and more precisely in the proportion of words which have been used exactly once, twice, three times, ... in the whole book. In this setting, Simon [20] pointed out the role of the probability mass function

$$
\rho \mathrm{B}(k, \rho+1), \quad k \geq 1,
$$

where B denotes the beta function and $\rho>0$ a parameter. He called (1) the Yule distribution, but nowadays (1) is rather referred to as the Yule-Simon distribution (with parameter $\rho$ ). In the present work, only the case $\rho=1 / p>1$ arises, although in the literature, $\rho$ may be any positive real number.

A well-known representation of the Yule-Simon distribution as a mixture of geometric distributions driven by the exponential of the negative of an exponential variable will be useful for us in the sequel. Namely, the change of variables $\mathrm{e}^{-t}=u$ in the integral below shows the identity

$$
\rho \mathrm{B}(k, \rho+1)=\rho \int_{0}^{\infty} \mathrm{e}^{-\rho t}\left(1-\mathrm{e}^{-t}\right)^{k-1} \mathrm{e}^{-t} \mathrm{~d} t, \quad k \geq 1 .
$$

We shall need an extension of Simon's result to processes, and in this direction, we introduce the following definition.

Definition 2.1 (Yule-Simon process). We call an integer-valued process on the unit time-interval, $Y=(Y(t))_{0 \leq t \leq 1}$, a Yule-Simon process with parameter $\rho>0$, if $Y$ is a time-inhomogeneous pure birth process started from $Y(0)=0$ a.s. and with time-dependent birth rates

$$
\lambda_{k}(t):=\lim _{h \rightarrow 0+} h^{-1} \mathbb{P}(Y(t+h)=k+1 \mid Y(t)=k) \quad \text { for } 0 \leq t<1 \text { and } k \in \mathbb{N}
$$

given by

$$
\lambda_{0}(t)=1 /(1-t) \quad \text { and } \quad \lambda_{k}(t)=k /(\rho t) \quad \text { for } k \geq 1 .
$$

In other words, a Yule-Simon process is a counting process ${ }^{2}$ which fulfills the time-inhomogeneous Markov property and has infinitesimal generator at time $t$

$$
\lim _{h \rightarrow 0+} h^{-1} \mathbb{E}[f(Y(t+h))-f(Y(t)) \mid Y(t)=k]= \begin{cases}(f(1)-f(0)) /(1-t) & \text { for } k=0, \\ (f(k+1)-f(k)) k /(\rho t) & \text { for } k \geq 1 .\end{cases}
$$

We first point at the following simple construction in terms of a standard Yule process (i.e. a pure birth process with unit birth rate per individual) taken in the logarithmic time.

\footnotetext{
${ }^{2}$ This means that $Y$ is a càdlàg integer-valued and non-decreasing process started from 0 and having only jumps of unit length a.s.
} 
Lemma 2.2. Let $Z=(Z(t))_{t \geq 0}$ be a standard Yule process started from $Z(0)=1$ a.s., and $U$ an independent uniform random variable on $[0,1]$. Set for every $t \in[0,1]$

$$
Y(t)= \begin{cases}0 & \text { if } t<U, \\ Z((\ln t-\ln U) / \rho) & \text { if } t \geq U .\end{cases}
$$

Then $Y=(Y(t))_{0 \leq t \leq 1}$ is a Yule-Simon process with parameter $\rho$.

Proof. The process $Y$ in the statement is plainly a time-inhomogeneous pure birth process started from 0 . By construction, its first hitting time of 1 occurs at time $U$, which has the uniform distribution on $[0,1]$. On the other hand, the probability that a Yule-Simon process is still at 0 at time $t \in(0,1)$ equals $\exp \left(-\int_{0}^{t}(1-s)^{-1} \mathrm{~d} s\right)=1-t$. Hence $Y$ has the same birth rate from the state 0 as a Yule-Simon process.

Next take any $k \geq 1$. The jump rate of a Yule process from $k$ to $k+1$ equals $k$, so after the logarithmic time change, the jump rate at time $t$ of $Y$ from $k$ to $k+1$ equals $k /(\rho t)$, that is that of a Yule-Simon process with parameter $\rho$. We conclude that $Y$ indeed satisfies the requirements of Definition 2.1.

As an immediate consequence, we can now justify our terminology.

Corollary 2.3. Let $Y=(Y(t))_{0 \leq t \leq 1}$ be a Yule-Simon process with parameter $\rho>0$. For every $0<t \leq 1$, we have:

(i) $\mathbb{P}[Y(t) \geq 1]=t$,

(ii) the conditional law of $Y(t)$ given $Y(t) \geq 1$ is the Yule-Simon distribution (1) with parameter $\rho$.

Proof. Using the construction of Lemma 2.2, we have $U=\inf \{t \geq 0: Y(t)=1\}$, which yields the first assertion. Then conditionally on $Y(t) \geq 1$, that is equivalently $U \leq t, U / t$ has the uniform distribution on $[0,1]$ and thus $(\ln t-\ln U) / \rho$ has the exponential distribution with parameter $\rho$. Recall that for every $r>0, Z(r)$ has the geometric law with parameter $\mathrm{e}^{-r}$, and that $Z$ is independent of $(\ln t-\ln U) / \rho$. The second assertion now follows from the representation (2) as a mixture of geometric distributions.

Remark 2.4. More generally, the combination of Lemma 2.2 with the fact that conditionally on $U \leq t, U / t$ has the uniform distribution on $[0,1]$, shows that conditionally on $Y(t) \geq 1$, the process $(Y(s t))_{0 \leq s \leq 1}$ is again a Yule-Simon process with the same parameter.

We conclude this section by recalling the asymptotic behavior

$$
\mathrm{B}(k, \rho+1) \sim \Gamma(\rho+1) k^{-(\rho+1)} \quad \text { as } k \rightarrow \infty ;
$$

This implies in particular that a Yule-Simon variable with parameter $\rho$ has finite moments of any order $r<\rho$, whereas its moment of order $\rho$ is infinite. For future use, we also record the following:

Corollary 2.5. Let $Y=(Y(t))_{0 \leq t \leq 1}$ be a Yule-Simon process with parameter $\rho$. Then:

(i) If $\rho>1$, then for every $t \in[0,1]$

$$
\mathbb{E}[Y(t)]=\frac{\rho}{\rho-1} t
$$

(ii) If $\rho>2$, then for every $0 \leq s \leq t \leq 1$

$$
\mathbb{E}[Y(t) Y(s)]=\frac{\rho^{2}}{(\rho-1)(\rho-2)} s\left(\frac{t}{s}\right)^{1 / \rho} .
$$

Proof. The first moment of the Yule-Simon law with parameter $\rho$ equals $\rho /(\rho-1)$ if $\rho>1$, and its variance equals $\rho^{2}(\rho-1)^{-2}(\rho-2)^{-1}$ if $\rho>2$. The formula (i) thus follows from Corollary 2.3, and further we have for the second moment

$$
\mathbb{E}\left[Y(t)^{2}\right]=\frac{\rho^{2}}{(\rho-1)(\rho-2)} t \quad \text { if } \rho>2 .
$$


On the other hand, a Yule process $Z$ grows exponentially in time and fulfills the branching property, so for every $k \geq 1$ and $0<s<t$, there is the identity

$$
\mathbb{E}[Z(t) \mid Z(s)=k]=k \mathbb{E}[Z(t-s)]=k \mathrm{e}^{t-s} .
$$

Thanks to Lemma 2.2, this yields

$$
\mathbb{E}[Y(t) \mid Y(s)=k]=\mathbb{E}[Z((\ln t-\ln U) / \rho) \mid Z((\ln s-\ln U) / \rho)=k]=k(t / s)^{1 / \rho} .
$$

The formula (ii) now follows from (4).

\subsection{Random series of Yule-Simon processes}

Next we turn our attention to convergence of certain series $\sum_{j} Y_{j} x_{j}$ of independent Yule-Simon processes, where the $x_{j}$ are random vectors in $\mathbb{R}^{d}$ that should be thought of as the sizes of the jumps made by some $d$-dimensional Lévy process during the unit time interval. The purpose of this section is to show that, under appropriate hypotheses and possibly after a proper compensation (i.e. centering), the sum of such a series does make sense. Although this is similar to the construction of Lévy processes, we shall see that integrability properties of Yule-Simon variables play a crucial role.

Specifically, we write $\mathbb{Q}$ for the distribution of the Yule-Simon process with some fixed parameter $\rho>0$, say on the space of counting functions on the unit time interval. Consider a Poisson point measure $\mathcal{N}$ with intensity $v \otimes \mathbb{Q}$, where $v$ is a Lévy measure on $\mathbb{R}^{d}$, in the sense that

$$
\int_{\mathbb{R}^{d}}\left(1 \wedge|x|^{2}\right) v(\mathrm{~d} x)<\infty
$$

and $|\cdot|$ stands for the Euclidean norm. The product form of this intensity measure allows us to assume that $\mathcal{N}$ is given in the form

$$
\mathcal{N}=\sum_{j} \delta_{\left(x_{j}, Y_{j}\right)}
$$

with $\sum_{j} \delta_{x_{j}}$ a Poisson point measure on $\mathbb{R}^{d}$ with intensity $v$, and $\left(Y_{j}\right)_{j \geq 1}$ an independent sequence of i.i.d. marks with law $\mathbb{Q}$ (i.e. a sequence of i.i.d. Yule-Simon processes).

We write for $0 \leq a<b \leq \infty$

$$
\mathcal{N}_{a, b}:=\sum_{j} \mathbb{1}_{\left\{a \leq\left|x_{j}\right|<b\right\}} \delta_{\left(x_{j}, Y_{j}\right)},
$$

which is again a Poisson point measure, now with intensity $v_{a, b} \otimes \mathbb{Q}$, where

$$
v_{a, b}(\mathrm{~d} x):=\mathbb{1}_{\{a \leq|x|<b\}} v(\mathrm{~d} x) .
$$

Recall from the superposition property of Poisson point measures that for every $k \geq 1$ and $0 \leq a_{1}<a_{2}<\cdots<a_{k} \leq \infty$, the Poisson point measures $\mathcal{N}_{a_{1}, a_{2}}, \mathcal{N}_{a_{2}, a_{3}}, \ldots, \mathcal{N}_{a_{k-1}, a_{k}}$ are independent.

For every $0<a<b \leq \infty, v_{a, b}$ is a finite measure and $\mathcal{N}_{a, b}$ possesses only finitely many atoms a.s. We can always define the step process

$$
\Sigma_{a, b}(t):=\sum_{j} \mathbb{1}_{\left\{a \leq\left|x_{j}\right|<b\right\}} Y_{j}(t) x_{j}, \quad t \in[0,1] .
$$

We assume henceforth that $\rho>1$, and observe also from Corollary 2.5(i) and Campbell's formula that for every $a \in(0,1]$ and $0 \leq t \leq 1$, there is the identity

$$
\mathbb{E}\left[\sum_{j} \mathbb{1}_{\left\{a \leq\left|x_{j}\right|<1\right\}} Y_{j}(t)\left|x_{j}\right|\right]=t \rho(\rho-1)^{-1} \int_{\{a \leq|x|<1\}}|x| v(\mathrm{~d} x) .
$$

So, if the Lévy measure fulfills

$$
\int_{\mathbb{R}^{d}}(1 \wedge|x|) v(\mathrm{~d} x)<\infty,
$$


which is a stronger requirement than (5), then $\Sigma_{0,1}(t)$ is a well-defined process given by a series that converges absolutely in $L^{1}(\mathbb{P})$, and $\Sigma(t):=\Sigma_{0,1}(t)+\Sigma_{1, \infty}(t)$ is an a.s. absolutely convergent series.

In the general case when the Lévy measure only fulfills (5), we use again Campbell's formula for $a \in(0,1]$ to compute the mean vector

$$
\mathbb{E}\left[\sum_{j} \mathbb{1}_{\left\{a \leq\left|x_{j}\right|<1\right\}} Y_{j}(t) x_{j}\right]=t \rho(\rho-1)^{-1} \int_{\{a \leq|x|<1\}} x v(\mathrm{~d} x)
$$

and define the compensated (or centered) sum

$$
\Sigma_{a, 1}^{(c)}(t):=\Sigma_{a, 1}(t)-t \rho(\rho-1)^{-1} \int_{\{a \leq|x|<1\}} x v(\mathrm{~d} x)
$$

To investigate the behavior of this quantity as $a \rightarrow 0+$, it is convenient to make the substitution $a=\mathrm{e}^{-r}$ with $r \geq 0$. For every $t \in(0,1]$, the process $\left(\Sigma_{\mathrm{e}^{-r}, 1}^{(c)}(t)\right)_{r \geq 0}$ has independent and centered increments, and is therefore a martingale. Whether or not this martingale converges as $r \rightarrow \infty$ depends crucially on the so-called Blumenthal-Getoor (upper) index of the Lévy measure $v$, which is defined as

$$
\beta(v):=\inf \left\{b>0: \int_{|x| \leq 1}|x|^{b} v(\mathrm{~d} x)<\infty\right\} .
$$

In particular, we always have $\beta(v) \leq 2$, and if (7) fails, then $\beta(v) \geq 1$. Further, in the stable case $v(\mathrm{~d} x)=|x|^{-\alpha-d} \mathrm{~d} x$ for some $0<\alpha<2$, one has plainly $\beta(\nu)=\alpha$.

Lemma 2.6. Suppose $\rho>1$ and take any $t \in(0,1]$.

(i) If $\beta(v)<\rho$, then the martingale $\left(\Sigma_{\mathrm{e}^{-r}, 1}^{(c)}(t)\right)_{r \geq 0}$ is uniformly integrable, and in particular the compensated sum $\Sigma_{a, 1}^{(c)}(t)$ converges a.s. as $a \rightarrow 0+$.

(ii) If $\beta(v)>\rho$, then a.s., $\Sigma_{a, 1}^{(c)}(t)$ does not converge as $a \rightarrow 0+$.

Proof. For the sake of simplicity, we shall establish first parts (i) and (ii) under the assumption that the function $r \mapsto$ $v\left(\left\{|x|>\mathrm{e}^{-r}\right\}\right)$ is continuous on $(0, \infty)$, i.e. that the Lévy measure $v$ gives no mass to spheres. In parts ( $\left.\mathrm{i}^{\prime}\right)$ and (ii') of this proof, we explain how this additional assumption can be removed.

(i) The martingale $\left(\Sigma_{\mathrm{e}^{-r}, 1}^{(c)}(t)\right)_{r \geq 0}$ is purely discontinuous with quadratic variation process $\sum_{0<s \leq r} \Delta_{s}^{2}$, where $\Delta_{s}$ denotes the norm of the (possible) jump at $s$. So $\Delta_{s}=Y_{j}(t)\left|x_{j}\right|$ if the Poisson measure $\mathcal{N}$ has an atom at $\left(x_{j}, Y_{j}\right)$ with $\left|x_{j}\right|=\mathrm{e}^{-s}$ (our standing assumption ensures that a.s., $\mathcal{N}$ possesses at most one such atom for any $s>0$ ), and $\Delta_{s}=0$ otherwise.

Suppose first that $\beta(v)<2$ and pick any $q \in(\beta(v) \vee 1, \rho \wedge 2)$. Recall from the Burkholder-Davis-Gundy inequality that this martingale is bounded in $L^{q}(\mathbb{P})$ if and only if

$$
\mathbb{E}\left[\left(\sum_{s \geq 0} \Delta_{s}^{2}\right)^{q / 2}\right]<\infty
$$

Since $q \leq 2$, there is basic inequality

$$
\left(\sum_{s \geq 0} \Delta_{s}^{2}\right)^{q / 2} \leq \sum_{s \geq 0}\left|\Delta_{s}\right|^{q}
$$

and we only need to check that

$$
\mathbb{E}\left[\sum_{j} \mathbb{1}_{\left\{\left|x_{j}\right|<1\right\}} Y_{j}(t)^{q}\left|x_{j}\right|^{q}\right]<\infty .
$$

In this direction, we get from Campbell's formula that

$$
\mathbb{E}\left[\sum_{j} \mathbb{1}_{\left\{\left|x_{j}\right|<1\right\}} Y_{j}(t)^{q}\left|x_{j}\right|^{q}\right]=\mathbb{E}\left[Y(t)^{q}\right] \int_{\{|x|<1\}}|x|^{q} v(\mathrm{~d} x) .
$$


Since $Y(t) \in L^{q}(\mathbb{P})$ (by (3) and Corollary 2.3, as $q<\rho$ ) and $\int_{\{|x|<1\}}|x|^{q} \nu(\mathrm{d} x)<\infty$ (from the definition of the Blumenthal-Getoor index, as $q>\beta(v))$, our claim is established.

Then suppose $\beta(v)=2<\rho$. Since $\int_{|x| \leq 1} x^{2} v(\mathrm{~d} x)<\infty$ anyway, we can perform the same calculation as above for $q=2$, and check that the martingale is bounded in $L^{2}(\mathbb{P})$.

(ii) Since $v$ gives no mass to spheres, it holds almost surely that for all radii $r>0$, the Poisson point measure $\mathcal{N}$ at most one atom $(x, Y)$ with $|x|=r$. Recall from (3) and Corollary 2.3 that

$$
\mathbb{Q}[Y(t)>1 /|x|] \sim t \Gamma(\rho+1)|x|^{\rho} \quad \text { as } x \rightarrow 0 .
$$

Our assumption $\beta(\nu)>\rho$ implies that $\int_{|x| \leq 1}|x|^{\rho} \nu(\mathrm{d} x)=\infty$, and it follows that $\mathcal{N}$ possesses a.s. infinitely many atoms $(x, Y)$ with $Y(t)|x|>1$ (but of course only finitely many atoms with $|x|>\mathrm{e}^{-r}$ ). As a consequence, the martingale $\left(\Sigma_{\mathrm{e}^{-r}, 1}^{(c)}(t)\right)_{r \geq 0}$ has infinitely many jumps of length greater than 1 a.s., and therefore cannot converge.

(i') The argument in (i) does not directly apply when the Lévy measure $v$ gives a positive mass to some spheres, because the Poisson measure $\mathcal{N}$ may then have several atoms on such spheres, and the quadratic variation process has thus a less handy expression. To circumvent this slight difficulty, recall that we have fixed some $q \in(\beta(\nu) \vee 1, \rho \wedge 2)$ when $\beta(v)<2$, and $q=2$ when $\beta(v)=2$.

The measure $\left(1 \wedge|x|^{q}\right) v(\mathrm{~d} x)$ is finite, and there is a sequence $\left(v_{n}\right)_{n \geq 1}$ of Lévy measures on $\mathbb{R}^{d}$ giving no mass to spheres, such that $\left(1 \wedge|x|^{q}\right) v_{n}(\mathrm{~d} x)$ converges weakly on $\mathbb{R}^{d}$ to $\left(1 \wedge|x|^{q}\right) v(\mathrm{~d} x)$ as $n \rightarrow \infty$. We can then construct (possibly on an enlarged probability space) a sequence of Poisson point measures $\mathcal{P}^{(n)}$ on $\mathbb{R}^{d}$ with intensity $v_{n}$, such that with probability $1,|x|^{q} \mathcal{P}^{(n)}(\mathrm{d} x)$ converges weakly to $|x|^{q} \mathcal{P}(\mathrm{d} x)$ as $n \rightarrow \infty$, where $\mathcal{P}$ is a Poisson point measure on $\mathbb{R}^{d}$ with intensity $v$. Introduce an independent sequence $\left(Y_{j}\right)_{j \geq 1}$ of i.i.d. Yule-Simon processes with law $\mathbb{Q}$, and for each fixed $n \geq 1$, enumerate the atoms of $\mathcal{P}^{(n)}$ in the decreasing order and mark them using the sequence $\left(Y_{j}\right)_{j \geq 1}$. This yields a Poisson point measure $\mathcal{N}^{(n)}$ with intensity $v_{n} \otimes \mathbb{Q}$, and then a compensated martingale $\left(\Sigma_{\mathrm{e}^{-r}, 1}^{(\mathrm{c}, n)}(t)\right)_{r \geq 0}$.

The Burkholder-Davis-Gundy inequality applied as in (i) shows that the preceding martingales are bounded in $L^{q}(\mathbb{P})$ uniformly for $n \geq 1$, namely

$$
\sup _{n \geq 1, r \geq 0} \mathbb{E}\left[\left|\Sigma_{\mathrm{e}^{-r}, 1}^{(\mathrm{c}, n)}(t)\right|^{q}\right]<\infty .
$$

On the other hand, it is plain from the construction that for each $r>0, \Sigma_{\mathrm{e}^{-r}, 1}^{(\mathrm{c}, n)}(t)$ converges a.s. as $n \rightarrow \infty$ towards a random variable which has the same distribution as $\Sigma_{\mathrm{e}^{-r}, 1}^{(\mathrm{c})}(t)$. By Fatou's lemma, the compensated martingale $\left(\Sigma_{\mathrm{e}^{-r}, 1}^{(\mathrm{c})}(t)\right)_{r \geq 0}$ is also bounded in $L^{q}(\mathbb{P})$, and thus converges a.s.

(ii') We decompose $v=v^{\prime}+v^{\prime \prime}$, where $v^{\prime}$ is a Lévy measure giving no mass to any sphere, and $v^{\prime \prime}$ a Lévy measure supported by an at most countable union of spheres. If $\int_{|x| \leq 1}|x|^{\rho} v^{\prime}(\mathrm{d} x)=\infty$, then the argument of (ii) applies exactly the same. Otherwise, we must have $\int_{|x| \leq 1}|x|^{\rho} \nu^{\prime \prime}(\mathrm{d} x)=\infty$, and there is thus a sequence $\left(r_{j}\right)_{j \geq 1}$ with $r_{j} \rightarrow \infty$ such that

$$
\sum_{j \geq 1} \mathrm{e}^{-\rho r_{j}} v\left(\left\{|x|=\mathrm{e}^{-r_{j}}\right\}\right)=\infty
$$

Write $\Delta_{j}$ (respectively, $\Delta_{j}^{(\mathrm{c})}$ ) for the jump of the process $\Sigma_{\mathrm{e}^{-} \cdot 1,1}(t)$ (respectively, of the compensated martingale $\left.\Sigma_{\mathrm{e}^{-} \cdot 1}^{(\mathrm{c})}(t)\right)$ at $r_{j}$, so $\Delta_{j}^{(\mathrm{c})}=\Delta_{j}-\mathbb{E}\left(\Delta_{j}\right)$. By construction, $\Delta_{j}$ is compound Poisson variable and the radial part of its Lévy measure is

$$
v\left(\left\{|x|=\mathrm{e}^{-r_{j}}\right\}\right) \mathbb{Q}\left[\mathrm{e}^{-r_{j}} Y(t) \in \cdot\right] .
$$

It follows from (10) that there is a constant $c>0$ (independent of $j$ ) such that

$$
\mathbb{P}\left[\left|\Delta_{j}^{(\mathrm{c})}\right|>1\right] \geq c\left(1 \wedge\left(v\left(\left\{|x|=\mathrm{e}^{-r_{j}}\right\}\right) \mathbb{Q}\left[Y(t)>\mathrm{e}^{r_{j}}\right]\right)\right),
$$

see for instance Corollary 1.3.2 in [11] and the discussion thereafter. We now see from (11) that $\sum_{j \geq 1} \mathbb{P}\left[\left|\Delta_{j}^{(\mathrm{c})}\right|>1\right]=\infty$, and since the variables $\Delta_{j}^{(\mathrm{c})}$ are independent (by the restriction property of Poisson random measures), we conclude from the Borel-Cantelli lemma that the compensated martingale has again infinitely many jumps with length greater than 1 a.s.

Remark 2.7. When the Lévy measure is symmetric, the compensation term is always zero and $\Sigma_{a, 1}(t)=\Sigma_{a, 1}^{(c)}(t)$ for all $a \in(0,1]$. Thus, if (7) fails and $\rho>\beta(v)$, then series $\sum Y_{j}(t) x_{j}$ is only semi-convergent, that is the limit $\lim _{a \rightarrow 0+} \sum \mathbb{1}_{\left\{a \leq\left|x_{j}\right|<1\right\}} Y_{j}(t) x_{j}$ exists in $\mathbb{R}^{d}$ although $\sum Y_{j}(t)\left|x_{j}\right|=\infty$ a.s. 
Throughout the rest of this work, we write $\Sigma_{0,1}^{(c)}(t)$ for the a.s. limit of $\Sigma_{a, 1}^{(c)}(t)$ as $a \rightarrow 0+$ whenever $\beta(v)<\rho$. We now compute the characteristic function of this process, and in this direction, we write $\theta \cdot x$ for the scalar product of two vectors $\theta$ and $x$ in $\mathbb{R}^{d}$.

Corollary 2.8. Let $\rho>1$ and $v$ be a Lévy measure with Blumenthal-Getoor index $\beta(v)<\rho$. Define for $\theta \in \mathbb{R}^{d}$

$$
\Phi_{1}(\theta):=\int_{|x| \geq 1}\left(1-\mathrm{e}^{\mathrm{i} \theta \cdot x}\right) v(\mathrm{~d} x) \quad \text { and } \quad \Phi_{0}^{(c)}(\theta):=\int_{|x|<1}\left(1-\mathrm{e}^{\mathrm{i} \theta \cdot x}+\mathrm{i} \theta \cdot x\right) v(\mathrm{~d} x) .
$$

The processes $\left(\Sigma_{1, \infty}(t)\right)_{0 \leq t \leq 1}$ and $\left(\Sigma_{0,1}^{(c)}(t)\right)_{0 \leq t \leq 1}$ are independent. Further, for all $k \geq 1, \theta_{1}, \ldots, \theta_{k} \in \mathbb{R}^{d}$ and $t_{1}, \ldots, t_{k} \in$ $[0,1]$, there are the identities

$$
\mathbb{E}\left[\exp \left\{\mathrm{i} \sum_{j=1}^{k} \theta_{j} \cdot \Sigma_{1, \infty}\left(t_{j}\right)\right\}\right]=\exp \left\{-\mathbb{E}\left[\Phi_{1}\left(\sum_{j=1}^{k} Y\left(t_{j}\right) \theta_{j}\right)\right]\right\}
$$

and

$$
\mathbb{E}\left[\exp \left\{\mathrm{i} \sum_{j=1}^{k} \theta_{j} \cdot \Sigma_{0,1}^{(c)}\left(t_{j}\right)\right\}\right]=\exp \left\{-\mathbb{E}\left[\Phi_{0}^{(c)}\left(\sum_{j=1}^{k} Y\left(t_{j}\right) \theta_{j}\right)\right]\right\},
$$

where $Y=(Y(t))_{0 \leq t \leq 1}$ denotes a Yule-Simon process with parameter $\rho$.

Proof. The claim that $\Sigma_{1, \infty}$ and $\Sigma_{0,1}^{(c)}$ are independent stems from the independence of the random measures $\mathcal{N}_{1, \infty}$ and $\mathcal{N}_{0,1}$. The expression for the characteristic function of $\Sigma_{1, \infty}$ follows from the construction of the latter and the formula for the Fourier functional of Poisson point measures. The same argument also yields a similar formula for the characteristic function of the compensated sum $\Sigma_{a, 1}^{(c)}$ for every $0<a<1$, namely

$$
\mathbb{E}\left[\exp \left\{\mathrm{i} \sum_{j=1}^{k} \theta_{j} \cdot \Sigma_{a, 1}^{(c)}\left(t_{j}\right)\right\}\right]=\exp \left\{-\mathbb{E}\left[\Phi_{a}^{(c)}\left(\sum_{j=1}^{k} Y\left(t_{j}\right) \theta_{j}\right)\right]\right\}
$$

with

$$
\Phi_{a}^{(c)}(\theta):=\int_{a \leq|x|<1}\left(1-\mathrm{e}^{\mathrm{i} \theta \cdot x}+\mathrm{i} \theta \cdot x\right) v(\mathrm{~d} x) .
$$

We then let $a \rightarrow 0+$, so $\Phi_{a}^{(c)}$ converges pointwise to $\Phi_{0}^{(c)}$. On the other hand, pick any $q \in(1 \vee \beta(v), \rho)$ and let $C:=\sup _{y \in \mathbb{R}}|y|^{-q}\left|1-\mathrm{e}^{\mathrm{i} y}+\mathrm{i} y\right|<\infty$, so that for all $a \in(0,1)$ and $\theta \in \mathbb{R}^{d}$,

$$
\left|\Phi_{a}^{(c)}(\theta)\right| \leq \int_{|x|<1}\left|1-\mathrm{e}^{\mathrm{i} \theta \cdot x}+\mathrm{i} \theta \cdot x\right| v(\mathrm{~d} x) \leq C|\theta|^{q} \int_{|x|<1}|x|^{q} v(\mathrm{~d} x) .
$$

The integral in the right-hand side is finite by (8); recall also that $\sum_{j=1}^{k} Y\left(t_{j}\right) \theta_{j} \in L^{q}(\mathbb{P})$. Our last assertion now follows from dominated convergence.

\subsection{Noise reinforced Lévy processes and their characteristic functions}

To start this section with, we consider the elephant random walk, that is the step reinforced random walk $\hat{S}=(\hat{S}(n))_{n \in \mathbb{N}}$ in the one-dimensional simple symmetric framework. It is known that $\hat{S}$ has a diffusive behavior when the memory parameter $p<1 / 2$, and more precisely, it has been shown in [2] that the rescaled process $\left(n^{-1 / 2} \hat{S}(\lfloor n t\rfloor)\right)_{t \geq 0}$ converges in distribution to the centered Gaussian process $\hat{B}=(\hat{B}(t))_{t \geq 0}$ with covariance

$$
\mathbb{E}[\hat{B}(s) \hat{B}(t)]=\frac{t^{p} s^{1-p}}{1-2 p} \quad \text { for } 0 \leq s \leq t .
$$

We also refer to $[3,7,8]$ for related works. 
We sketch here a quick proof for this fact which may be of independent interest. It is readily seen that the elephant random walk is a time-inhomogeneous Markov chain on $\mathbb{Z}$. Specifically, the conditional probability that its $(n+1)$ th increment is $\hat{X}_{n+1}=1$ given the trajectory up to time $n,(\hat{S}(j))_{0 \leq j \leq n}$, obviously equals

$$
(1-p) / 2+p(1 / 2+\hat{S}(n) / 2 n)=1 / 2+p \hat{S}(n) / 2 n
$$

and the conditional probability that $\hat{X}_{n+1}=-1$ equals $1 / 2-p \hat{S}(n) / 2 n$. By classical diffusion-approximation techniques (see e.g. Section 7.4 in Ethier and Kurtz [12]) we deduce that $n^{-1 / 2} \hat{S}(\lfloor n t\rfloor)$ converges to the time-inhomogeneous diffusion with infinitesimal generator at time $t$ given by $\mathcal{G}_{t} f(x)=\frac{1}{2} f^{\prime \prime}(x)+p x t^{-1} f^{\prime}(x)$. We can also represent the limit process as the solution to the stochastic differential equation

$$
\mathrm{d} \hat{B}(t)=\mathrm{d} B(t)+p t^{-1} \hat{B}(t) \mathrm{d} t
$$

where $B=(B(t))_{t \geq 0}$ denotes a standard linear Brownian motion. One can solve this first-order linear differential equation explicitly and get

$$
\hat{B}(t)=t^{p} \int_{0}^{t} s^{-p} \mathrm{~d} B(s), \quad t \geq 0
$$

(observe that the stochastic integral only makes sense for $p<1 / 2$ ), from which it is seen that $\hat{B}$ is indeed a centered Gaussian process with covariance given by (12). We stress that this argument only works in this very situation, the Markov property fails for step reinforced random walks in higher dimension, or when the steps are not simple.

We call a centered Gaussian process with covariance (12) a noise reinforced linear Brownian motion, and more generally in higher dimension $d \geq 1$, we call noise reinforced Brownian motion with memory parameter $p$ a process in $\mathbb{R}^{d}$ whose coordinates are given by $d$ independent noise reinforced linear Brownian motions. The following lemma points at another relation with Yule-Simon processes which will be important for us.

Lemma 2.9. Let $M$ be a $d \times d$ matrix, and $\hat{B}$ a noise reinforced Brownian motion in $\mathbb{R}^{d}$ with memory parameter $p<1 / 2$. Then for all $k \geq 1, \theta_{1}, \ldots, \theta_{k} \in \mathbb{R}^{d}$ and $t_{1}, \ldots, t_{k} \in[0,1]$, we have

$$
\mathbb{E}\left[\exp \left\{\mathrm{i} \sum_{j=1}^{k} \theta_{j} \cdot M \hat{B}\left(t_{j}\right)\right\}\right]=\exp \left\{-\frac{1-p}{2} \mathbb{E}\left[\mathbf{q}\left(\sum_{j=1}^{k} Y\left(t_{j}\right) \theta_{j}\right)\right]\right\},
$$

where $\mathbf{q}$ denotes the positive semi-definite quadratic form on $\mathbb{R}^{d}$ induced by $M$, i.e. $\mathbf{q}(x)=|M x|^{2}$.

Proof. We first consider the case of dimension $d=1$, so the $\theta_{i}$ are reals and we may further assume that $M=1$. We deduce from (12) that the variable $\sum_{j=1}^{k} \theta_{j} \hat{B}\left(t_{j}\right)$ is Gaussian with covariance

$$
\frac{1}{1-2 p} \sum_{j, \ell=1}^{k} \theta_{j} \theta_{\ell}\left(t_{j} \vee t_{\ell}\right)^{p}\left(t_{j} \wedge t_{\ell}\right)^{1-p}
$$

Comparing with Corollary 2.5(ii), we can express the above quantity as

$$
(1-p) \mathbb{E}\left[\left(\sum_{j=1}^{k} Y\left(t_{j}\right) \theta_{j}\right)^{2}\right]
$$

and hence

$$
\mathbb{E}\left[\exp \left\{\mathrm{i} \sum_{j=1}^{k} \theta_{j} \hat{B}\left(t_{j}\right)\right\}\right]=\exp \left\{-\frac{1-p}{2} \mathbb{E}\left[\left(\sum_{j=1}^{k} Y\left(t_{j}\right) \theta_{j}\right)^{2}\right]\right\} .
$$

In dimension $d \geq 1$, we then obtain the formula of the statement when $M=\operatorname{Id}_{d}$ is the identity matrix and thus $\mathbf{q}$ is the square of the Euclidean norm, by using the independence of the coordinates. Finally, the case when $M$ is an arbitrary $d \times d$ matrix follows, writing $\theta_{j} \cdot M \hat{B}\left(t_{j}\right)=M^{T} \theta_{j} \cdot \hat{B}\left(t_{j}\right)$. 
We next briefly recall the Lévy-Itô construction of Lévy processes in $\mathbb{R}^{d}$. Consider a positive semi-definite quadratic form $\mathbf{q}$ on $\mathbb{R}^{d}, a \in \mathbb{R}^{d}$, and $\Lambda$ a Lévy measure on $\mathbb{R}^{d}$. Let $M$ be any $d \times d$ matrix such that $|M x|^{2}=\mathbf{q}(x), B$ a $d$ dimensional Brownian motion, and $\mathcal{M}(\mathrm{d} t, \mathrm{~d} x)$ a Poisson random measure on $[0, \infty) \times \mathbb{R}^{d}$ with intensity $\mathrm{d} t \times \Lambda(\mathrm{d} x)$ which is independent of $B$. Then, the process $\xi=(\xi(t))_{t \geq 0}$ defined by

$$
\xi(t)=M B(t)+t a+\int_{[0, t] \times \mathbb{R}^{d}} x \mathbb{1}_{\{|x| \geq 1\}} \mathcal{M}(\mathrm{d} s, \mathrm{~d} x)+\int_{[0, t] \times \mathbb{R}^{d}} x \mathbb{1}_{\{|x|<1\}} \mathcal{M}^{(c)}(\mathrm{d} s, \mathrm{~d} x),
$$

where the second stochastic integral with the notation $\mathcal{M}^{(c)}$ refers to the compensated version of Poissonian integral, is a Lévy process with characteristics $(\mathbf{q}, a, \Lambda)$. The characteristic exponent of $\xi$ is given by the Lévy-Khintchin formula

$$
\Psi(\theta):=\frac{1}{2} \mathbf{q}(\theta)-\mathrm{i} a \cdot \theta+\int_{\mathbb{R}^{d}}\left(1-\mathrm{e}^{\mathrm{i} \theta \cdot x}+\mathrm{i} \theta \cdot x \mathbb{1}_{\{|x|<1\}}\right) \Lambda(\mathrm{d} x), \quad \theta \in \mathbb{R}^{d},
$$

and we have $\mathbb{E}\left[\mathrm{e}^{\mathrm{i} \theta \cdot \xi(t)}\right]=\exp \{-t \Psi(\theta)\}$. Further, the symmetric bilinear form associated to $\mathbf{q}$ is the covariance matrix of the Gaussian component $M B$ of $\xi$; recall also that the sample paths of $\xi$ have finite variations a.s. if and only if $\mathbf{q}=0$ and $\int_{|x| \leq 1}|x| \Lambda(\mathrm{d} x)<\infty$.

We will now define the noise reinforced version $\hat{\xi}$ of $\xi$. In this direction, recall from the preceding section that $\mathbb{Q}$ stands for the law of a Yule-Simon process with parameter $\rho=1 / p$, and the notation for random series of Yule-Simon processes. Recall also from (8) that $\beta(v)$ stands for the Blumenthal-Getoor index of a Lévy measure $v$, and define the Blumenthal-Getoor index of a Lévy process $\xi$ with characteristics $(\mathbf{q}, a, \Lambda)$ as

$$
\beta= \begin{cases}\beta(\Lambda) & \text { if } \mathbf{q}=0 \\ 2 & \text { if } \mathbf{q} \neq 0\end{cases}
$$

We then say that $p \in(0,1)$ is an admissible memory parameter for $(\mathbf{q}, a, \Lambda)$ if $p \beta<1$.

Definition 2.10 (Noise reinforced Lévy process). Let $\mathbf{q}$ be a positive semi-definite quadratic form on $\mathbb{R}^{d}, a \in \mathbb{R}^{d}, \Lambda$ a Lévy measure on $\mathbb{R}^{d}$ and $p \in\left(0,1 \wedge \beta^{-1}\right)$ an admissible memory parameter for $(\mathbf{q}, a, \Lambda)$. Let $\mathcal{N}=\sum \delta_{\left(x_{j}, Y_{j}\right)}$ be a Poisson point measure with intensity $v \otimes \mathbb{Q}$, where $v=(1-p) \Lambda$. In the case $\mathbf{q} \neq 0$, let further $M$ be any $d \times d$ matrix such that $|M x|^{2}=\mathbf{q}(x)$ and $\hat{B}$ a $d$-dimensional noise reinforced Brownian motion with memory parameter $p$, which is independent of $\mathcal{N}$.

We now define a process $\hat{\xi}=(\hat{\xi}(t))_{0 \leq t \leq 1}$ by

$$
\hat{\xi}(t):=M \hat{B}(t)+t a+\Sigma_{1, \infty}(t)+\Sigma_{0,1}^{(c)}(t),
$$

where $\Sigma_{1, \infty}(t)$ is defined in $(6), \Sigma_{0,1}^{(c)}(t)$ is constructed in Lemma 2.6, and in the case $\mathbf{q}=0$, the term $M \hat{B}(t)$ should be interpreted as 0 .

We call $\hat{\xi}$ a noise reinforced Lévy process with characteristics $(\mathbf{q}, a, \Lambda, p)$.

It may be worthwhile to comment a bit on this definition. The construction bears obvious similarities with the Lévy-Itô decomposition, replacing Brownian motion by its reinforced version, and the role of Poisson processes being played by Yule-Simon processes. The sizes of the jumps made by the Lévy process $\xi$ during the unit time interval form a Poisson point process with intensity $\Lambda$, and roughly speaking, the effect of the reinforcement with memory parameter $p$ implies that each such jump is erased with probability $p$, independently one from the other. This corresponds to a $(1-p)$ thinning, and hence, if we consider the family of jumps of the reinforced process and disregard their multiplicities (which correspond to repetitions of the same jump), one gets a Poisson point measure with intensity $v=(1-p) \Lambda$. Plainly, step reinforcement has no effects on a pure drift process $t \mapsto a t$, which explains why, contrary to the Lévy measure, the drift coefficient remains unchanged. Note also that in general, the repetition of certain jumps not only impedes the Markov property of noise reinforced Lévy processes, but also entails that the distribution of $\hat{\xi}$ is singular with respect to that of $\xi$.

We now arrive at the following formula for the characteristic function of a noise reinforced Lévy process.

Corollary 2.11. Let $\hat{\xi}$ be a noise reinforced Lévy process with characteristics $(\mathbf{q}, a, \Lambda, p)$. Then for all $k \geq 1, \theta_{1}, \ldots, \theta_{k} \in$ $\mathbb{R}^{d}$ and $t_{1}, \ldots, t_{k} \in[0,1]$, we have

$$
\mathbb{E}\left[\exp \left\{\mathrm{i} \sum_{j=1}^{k} \theta_{j} \cdot \hat{\xi}\left(t_{j}\right)\right\}\right]=\exp \left\{-(1-p) \mathbb{E}\left[\Psi\left(\sum_{j=1}^{k} Y\left(t_{j}\right) \theta_{j}\right)\right]\right\},
$$


where $\Psi$ denotes the characteristic exponent (13) of a Lévy process $\xi$ with characteristics $(\mathbf{q}, a, \Lambda)$, and $Y=(Y(t))_{0 \leq t \leq 1}$ a Yule-Simon process with parameter $\rho=1 / p$.

Proof. Since $v=(1-p) \Lambda$, in the notation of Corollary 2.8 , there is the identity

$$
(1-p) \Psi(\theta)=\frac{1-p}{2} \mathbf{q}(\theta)-\mathrm{i}(1-p) a \cdot \theta+\Phi_{1}(\theta)+\Phi_{0}^{(c)}(\theta), \quad \theta \in \mathbb{R}^{d}
$$

Our claim is now plain from the construction of $\hat{\xi}$, by combining Corollary 2.8, Lemma 2.9 and Corollary 2.5(i).

For instance, we deduce from Corollary 2.5(i) and Corollary 2.11 that if $\xi$ is a standard Cauchy process, i.e. with characteristic exponent $\Psi(\theta)=|\theta|$, then every memory parameter $p \in(0,1)$ is admissible and for every $t \in(0,1], \hat{\xi}(t)$ has the Cauchy distribution with scale parameter $t$ (and thus $\hat{\xi}$ has the same one-dimensional marginals as $\xi$ ).

To conclude this section, we point at a few simple properties that noise reinforced Lévy processes inherit from usual Lévy processes, and which can be seen either directly from the construction in Definition 2.10, or easily checked from Corollary 2.11 (and hence proofs are omitted).

\section{Proposition 2.12.}

(i) [Additivity property] Let $\hat{\xi}$ and $\hat{\xi}^{\prime}$ be two independent noise reinforced Lévy processes with respective characteristics $(\mathbf{q}, a, \Lambda, p)$ and $\left(\mathbf{q}^{\prime}, a^{\prime}, \Lambda^{\prime}, p\right)$. The sum $\hat{\xi}+\hat{\xi}^{\prime}$ is then a noise reinforced Lévy process with characteristics $(\mathbf{q}+$ $\left.\mathbf{q}^{\prime}, a+a^{\prime}, \Lambda+\Lambda^{\prime}, p\right)$.

(ii) [Independence of coordinates] The coordinates $\left(\hat{\xi}_{1}, \ldots, \hat{\xi}_{d}\right)$ of a d-dimensional noise reinforced Lévy process $\hat{\xi}$ are independent if and only if the characteristic exponent $\Psi$ has the form

$$
\Psi(\theta)=\Psi_{1}\left(\theta_{1}\right)+\cdots+\Psi_{d}\left(\theta_{d}\right), \quad \theta=\left(\theta_{1}, \ldots, \theta_{d}\right) \in \mathbb{R}^{d},
$$

that is equivalently, if and only if the coordinates $\left(\xi_{1}, \ldots, \xi_{d}\right)$ of the d-dimensional Lévy process $\xi$ are independent.

(iii) [Stability] Suppose that the Lévy process $\xi$ is (strictly) stable with index $\alpha \in(0,2]$, that is its characteristic exponent fulfills $\Psi(c \theta)=c^{\alpha} \Psi(\theta)$ for all $\theta \in \mathbb{R}^{d}$ and $c>0$. Then the memory parameter $p \in(0,1)$ is admissible if and only if $p \alpha<1$, and in that case, the noise reinforced version $\hat{\xi}$ is an $\alpha$-stable process, in the sense that its finite dimensional marginals follow $\alpha$-stable laws.

Remark 2.13. Statements (i) and (ii) might come as a surprise, as in general, the sum of two independent step-reinforced random walks is not a step-reinforced random walk, and likewise, step reinforcement does not preserve the independence of coordinates for random walks.

\section{Noise reinforcement as limit of step reinforcements}

We now have all the ingredients needed to state the main result of this work. Recall from the introduction the definition of the step reinforced version $\hat{S}$ of a random walk $S$ for a given memory parameter $p \in(0,1)$.

Theorem 3.1. Let $\xi=(\xi(t))_{t \geq 0}$ be a Lévy process with characteristics $(\mathbf{q}, a, \Lambda)$. For every $n \geq 1$, we write $S^{(n)}=$ $(\xi(k / n))_{k \geq 0}$ for the discrete time skeleton of $\xi$ with time-mesh $1 / n$. Let $p \in(0,1)$ be an admissible memory parameter for $(\mathbf{q}, a, \Lambda)$ and let $\hat{S}^{(n)}=\left(\hat{S}^{(n)}(k)\right)_{k \geq 0}$ denote the step reinforced random walk with memory parameter $p$.

Then as $n \rightarrow \infty$, there is the weak convergence in the sense of finite dimensional distributions

$$
\left(\hat{S}^{(n)}(\lfloor t n\rfloor)\right)_{0 \leq t \leq 1} \Longrightarrow(\hat{\xi}(t))_{0 \leq t \leq 1},
$$

where $\hat{\xi}$ denotes a noise reinforced Lévy process with characteristics $(\mathbf{q}, a, \Lambda, p)$.

Remark 3.2. In the case when $\xi$ is an isotropic stable Lévy process, Theorem 3.1 bears a close relation to Theorem 2 in [5] and its multi-dimensional extension in [6]. Specifically, Businger established the weak convergence in the sense of finite dimensional distributions of $\hat{S}^{(n)}(\lfloor\cdot n\rfloor)$ towards some non-Lévy stable process that is specified via its characteristic function; a representation as an integral of an $\alpha$-stable random measure is also given. Here, we further identify this limit as a noise reinforced (stable) Lévy process $\hat{\xi}$. Although the present approach also relies on establishing the convergence of characteristic functions, our proof based on propagation of chaos techniques differs much from the tedious estimates in [5] and [6] which are specific to the stable case. 
The rest of this section is devoted to the proof of Theorem 3.1, and we shall first connect step reinforcement and Yule-Simon processes asymptotically.

Recall that $X_{1}, X_{2}, \ldots$ is an i.i.d. sequence of random variables, and $\varepsilon_{2}, \varepsilon_{3}, \ldots$ an independent i.i.d. sequence of Bernoulli variables with parameter $p$. We would like to view $X_{1}, X_{2}, \ldots$ as a sequence of distinct words; beware however that when their common distribution has an atom, then $\mathbb{P}\left[X_{1}=X_{2}\right]>0$. This is only a minor problem that is easily overcome by introducing just for this purpose a further i.i.d. sequence $U_{1}, U_{2}, \ldots$ of uniform random variables which is independent of the preceding. So writing $X_{j}^{\prime}=\left(X_{j}, U_{j}\right)$ for every $j \geq 1$, the variables $X_{1}^{\prime}, X_{2}^{\prime}, \ldots$ are now all different a.s. and can be thought of as a sequence of distinct words. Then viewing each variable $X_{k}^{\prime}$ for which $\varepsilon_{k}=0$ as a new word that never occurred previously, the sequence $\hat{X}_{1}^{\prime}, \hat{X}_{2}^{\prime}, \ldots$ of the increments of the step reinforced random walk that was described in the introduction follows precisely Simon's model that has been recalled in Section 2 . We introduce the counting processes for occurrences, namely we set for all integers $j, k \geq 1$

$$
N_{j}(k):=\operatorname{Card}\left\{1 \leq \ell \leq k: \hat{X}_{\ell}^{\prime}=X_{j}^{\prime}\right\},
$$

so that the step reinforced random walk can be expressed in the form

$$
\hat{S}(k)=\sum_{j=1}^{\infty} N_{j}(k) X_{j} .
$$

Note that when $\varepsilon_{j}=1$, an event which has probability $p, X_{j}$ will never be used as a word and $N_{j}(k)=0$ for all $k \geq 1$, whereas when $\varepsilon_{j}=0$, one has $N_{j}(k)=0$ for $k<j$ and $N_{j}(k) \geq 1$ for $k \geq j$.

The main purpose of this section is to point out that the Yule-Simon process arises in the limit of the empirical distribution of (time-rescaled versions of) the counting processes. In this direction, we consider a complex valued functional $F$ on the space of càdlàg paths $\omega:[0,1] \rightarrow \mathbb{N}$, which is continuous for Skorohod's topology. For simplicity, we further assume that $F(0)=0$, where by a slightly abusive notation, we denote the path identically zero by 0 .

Proposition 3.3. If $F$ is bounded, then there is the convergence in probability

$$
\lim _{n \rightarrow \infty} \frac{1}{n} \sum_{j=1}^{n} F\left(N_{j}(\lfloor\cdot n\rfloor)\right)=(1-p) \mathbb{E}[F(Y)],
$$

where we use the notation $N_{j}(\lfloor\cdot n\rfloor)$ for the time-rescaled counting process $\left(N_{j}(\lfloor\text { tn }\rfloor)\right)_{0 \leq t \leq 1}$ and $Y=(Y(t))_{0 \leq t \leq 1}$ denotes a Yule-Simon process with parameter $\rho=1 / p$

Remark 3.4. The result of Simon [20] corresponds to the case when the functional $F$ only depends on the terminal value of the path.

We will prove that the stated convergence holds in $L^{2}(\mathbb{P})$ (which, in the present framework, is equivalent to convergence in probability, since $F$ is bounded), by establishing the so-called propagation of chaos. This requires estimating first and second moments, and the calculations rely on the following lemma.

Lemma 3.5. For every $n \geq 1$, let $u(n)$ and $v(n)$ denote two independent uniform sample from $[n]:=\{1, \ldots, n\}$, which are further independent of the sequences $\left(X_{j}\right)_{j \geq 1}$ and $\left(\varepsilon_{i}\right)_{i \geq 2}$. We have as $n \rightarrow \infty$ :

(i) the conditional distribution of the time-rescaled counting process $N_{u(n)}(\lfloor\cdot n\rfloor)$ given $\varepsilon_{u(n)}=0$, converges in the sense of Skorohod towards the law of a Yule-Simon process with parameter $\rho=1 / p$,

(ii) the conditional distribution of the pair of time-rescaled counting processes

$$
\left(N_{u(n)}(\lfloor\cdot n\rfloor), N_{v(n)}(\lfloor\cdot n\rfloor)\right)
$$

given $\varepsilon_{u(n)}=\varepsilon_{v(n)}=0$, converges in the sense of Skorohod towards the law of a pair of independent Yule-Simon processes with parameter $1 / p$.

Proof. (i) Consider a sequence $(j(n))_{n \geq 1}$ with $j(n) / n \rightarrow u \in(0,1)$. Fix $n \geq 1$ and work conditionally on $\varepsilon_{j}(n)=0$. The counting process $\left(N_{j(n)}(\ell)\right)_{\ell \geq 0}$ is a time inhomogeneous Markov chain on $\mathbb{N}$ whose sample paths are first deterministic 
up to time $j(n)$ with $N_{j(n)}(\ell)=0$ for $\ell<j(n)$ and $N_{j(n)}(j(n))=1$. Then for any $\ell \geq j(n)$ and $a$ positive integer,

$$
\begin{aligned}
& \mathbb{P}\left[N_{j(n)}(\ell+1)=a+1 \mid N_{j(n)}(\ell)=a\right]=p a / \ell, \\
& \mathbb{P}\left[N_{j(n)}(\ell+1)=a \mid N_{j(n)}(\ell)=a\right]=1-p a / \ell .
\end{aligned}
$$

As a consequence, we have for any $u \leq t<t^{\prime}$ and any $a$ positive integer:

$$
\mathbb{P}\left[N_{j(n)}\left(\left\lfloor t^{\prime} n\right\rfloor\right)=a \mid N_{j(n)}(\lfloor t n\rfloor)=a\right]=\prod_{\ell=\lfloor t n\rfloor}^{\left\lfloor t^{\prime} n\right\rfloor-1}(1-a p \ell) .
$$

When $n \rightarrow \infty$, the quantity above converges to

$$
\exp \left(-a p \int_{t}^{t^{\prime}} s^{-1} \mathrm{~d} s\right)=\left(t / t^{\prime}\right)^{a p} .
$$

By considering the waiting times between the hitting times of consecutive positive integers and applying the Markov property, we now see that the sequence of time-rescaled counting processes $\left.N_{j(n)}(\lfloor\cdot n\rfloor)\right)$ converges in distribution as $n \rightarrow$ $\infty$ to a time-inhomogeneous pure birth process started from 0 , that hits 1 first at time $u$, and whose jump rate at time $s \geq u$ from $a \geq 1$ to $a+1$ equals to $a p / s$. This is a description of the time-change Yule process $\left(\mathbb{1}_{\{u \leq t\}} Z(p(\ln t-\ln u))\right)_{t \geq 0}$.

Now it suffices to replace the deterministic sequence $(j(n))_{n \geq 1}$ by the random sequence $(u(n))_{n \geq 1}$, and observe that then $u(n) / n$ converges in law to a uniform random variable on $(0,1)$. The representation of the Yule-Simon process in Lemma 2.2 concludes the proof of our claim.

(ii) The proof follows the same path as (i) with heavier notation. Consider two sequences $(j(n))_{n \geq 1}$ and $(k(n))_{n \geq 1}$ such that $j(n) / n \rightarrow u$ and $k(n) / n \rightarrow v$, where we first suppose that $0<u<v<1$. We start by fixing $n$ sufficiently large so $1 \leq j(n)<k(n)<n$, and work conditionally on $\varepsilon_{j(n)}=\varepsilon_{k(n)}=0$.

The pair of counting processes $\mathbf{N}=\left(N_{j(n)}(\ell), N_{k(n)}(\ell)\right)_{\ell \geq 0}$ is a time inhomogeneous Markov chain on $\mathbb{N}^{2}$ whose transitions can be computed explicitly. Specifically, the path is first deterministic up to time $j(n)$ with

$$
\mathbf{N}(\ell)= \begin{cases}(0,0) & \text { for } \ell<j(n), \\ (1,0) & \text { for } \ell=j(n) .\end{cases}
$$

Then for $j(n) \leq \ell<k(n)-1$ and $a$ positive integer,

$$
\begin{aligned}
& \mathbb{P}[\mathbf{N}(\ell+1)=(a+1,0) \mid \mathbf{N}(\ell)=(a, 0)]=p a / \ell, \\
& \mathbb{P}[\mathbf{N}(\ell+1)=(a, 0) \mid \mathbf{N}(\ell)=(a, 0)]=1-p a / \ell .
\end{aligned}
$$

Since $\varepsilon_{k(n)}=0$, the transition at the $k(n)$ th step is again deterministic with

$$
\mathbf{N}(k(n))-\mathbf{N}(k(n)-1)=(0,1) .
$$

Finally, for $\ell \geq k(n)$ and $a, b$ positive integers with $a+b \leq \ell$, one has

$$
\begin{aligned}
& \mathbb{P}[\mathbf{N}(\ell+1)=(a+1, b) \mid \mathbf{N}(\ell)=(a, b)]=p a / \ell, \\
& \mathbb{P}[\mathbf{N}(\ell+1)=(a, b+1) \mid \mathbf{N}(\ell)=(a, b)]=p b / \ell, \\
& \mathbb{P}[\mathbf{N}(\ell+1)=(a, b) \mid \mathbf{N}(\ell)=(a, b)]=1-p(a+b) / \ell .
\end{aligned}
$$

Analyzing the jump chain and holding times as in (i), one deduces that under the conditional law given $\varepsilon_{j(n)}=\varepsilon_{k(n)}=$ 0 , the distribution of the pair of time-rescaled counting processes $\left(N_{j(n)}(\lfloor\cdot n\rfloor), N_{k(n)}(\lfloor\cdot n\rfloor)\right)$ converges in the sense of Skorohod to that of

$$
\left(\mathbb{1}_{\{u \leq t\}} Z(p(\ln t-\ln u)), \mathbb{1}_{\{v \leq t\}} Z^{\prime}(p(\ln t-\ln v))\right)_{t \geq 0},
$$

where $Z$ and $Z^{\prime}$ denote two independent Yule process both started from 1. Alternatively, one may also consider the asymptotic behavior of the transition probabilities above, compare them with the infinitesimal generator of a pair of independent Yule processes, and invoke a general result on approximation of Feller processes by discrete time Markov chains (see, e.g. Theorem 19.28 in Kallenberg [13]). 
That the same holds in the case $0<v<u<1$ is clear by symmetry. Since the pair $(u(n) / n, v(n) / n)$ converges in law towards a pair of independent uniform random variables on $[0,1]$ and $\mathbb{P}\left[\varepsilon_{u(n)}=0, \varepsilon_{v(n)}=0 \mid u(n), v(n)\right]=(1-p)^{2}$ except when $u(n)=1$, or $v(n)=1$, or $u(n)=v(n)$, an event whose probability tends to 0 as $n \rightarrow \infty$, our claim now follows readily from the representation of the Yule-Simon process in Lemma 2.2.

Proof of Proposition 3.3. Since $N_{j}(\lfloor\cdot n\rfloor)=0$ when $\varepsilon_{j}=1$ and $F(0)=0$, we have

$$
\frac{1}{n} \sum_{j=1}^{n} F\left(N_{j}(\lfloor\cdot n\rfloor)\right)=\frac{1}{n} \sum_{j=1}^{n} \mathbb{1}_{\left\{\varepsilon_{j}=0\right\}} F\left(N_{j}(\lfloor\cdot n\rfloor)\right) .
$$

We deduce from Lemma 3.5(i) that

$$
\begin{aligned}
\lim _{n \rightarrow \infty} \mathbb{E}\left[\frac{1}{n} \sum_{j=1}^{n} \mathbb{1}_{\left\{\varepsilon_{j}=0\right\}} F\left(N_{j}(\lfloor\cdot n\rfloor)\right)\right] & =\lim _{n \rightarrow \infty} \mathbb{E}\left[\mathbb{1}_{\left\{\varepsilon_{u(n)}=0\right\}} F\left(N_{u(n)}(\lfloor\cdot n\rfloor)\right)\right] \\
& =(1-p) \mathbb{E}[F(Y)] .
\end{aligned}
$$

Similarly, we deduce from Lemma 3.5(ii) that

$$
\begin{aligned}
& \lim _{n \rightarrow \infty} \mathbb{E}\left[\left(\frac{1}{n} \sum_{j=1}^{n} \mathbb{1}_{\left\{\varepsilon_{j}=0\right\}} F\left(N_{j}(\lfloor\cdot n\rfloor)\right)\right)^{2}\right] \\
& =\lim _{n \rightarrow \infty} \mathbb{E}\left[\frac{1}{n^{2}} \sum_{j, k=1}^{n} \mathbb{1}_{\left\{\varepsilon_{j}=0\right\}} F\left(N_{j}(\lfloor\cdot n\rfloor)\right) \mathbb{1}_{\left\{\varepsilon_{k}=0\right\}} F\left(N_{k}(\lfloor\cdot n\rfloor)\right)\right] \\
& =\lim _{n \rightarrow \infty} \mathbb{E}\left[\mathbb{1}_{\left\{\varepsilon_{u(n)}=0\right\}} F\left(N_{u(n)}(\lfloor\cdot n\rfloor)\right) \mathbb{1}_{\left\{\varepsilon_{v(n)}=0\right\}} F\left(N_{v(n)}(\lfloor\cdot n\rfloor)\right)\right] \\
& =(1-p)^{2} \mathbb{E}[F(Y)]^{2},
\end{aligned}
$$

which completes the proof.

Our next goal it to extend Proposition 3.3 to unbounded functionals, and this relies on moment bounds for the counting processes $N_{j}$.

Lemma 3.6. For every $\gamma \in(1,1 / p)$, there exist numerical constants $c=c(\gamma, p)>0$ and $\eta=\eta(\gamma, p) \in(0,1)$ such that

$$
\mathbb{E}\left[N_{j}(n)^{\gamma}\right] \leq c(n / j)^{\eta} \quad \text { for all } 1 \leq j \leq n .
$$

Proof. We first observe from Jensen's inequality, that working with some $\gamma^{\prime} \in(\gamma, 1 / p)$ in place of $\gamma$, it suffices to establish the inequality in the statement with $\eta=1$. We shall show that one can pick a constant $c>0$ sufficiently large, such that for every $j \geq 1$, the process $\left(c+N_{j}(k)\right)^{\gamma} / k$ for $k \geq j$ is a supermartingale. As a consequence, we have

$$
\mathbb{E}\left[\left(c+N_{j}(n)\right)^{\gamma}\right] \leq(c+1)^{\gamma} n / j,
$$

which thus proves our claim.

The conditional probability that $N_{j}(k+1)=\ell+1$ given $N_{j}(k)=\ell$ equals $p \ell / k$ for any $j+\ell \leq k+1$, and therefore

$$
\begin{aligned}
\mathbb{E} & {\left[\frac{\left(c+N_{j}(k+1)\right)^{\gamma}}{k+1}-\frac{\left(c+N_{j}(k)\right)^{\gamma}}{k} \mid N_{j}(k)=\ell\right] } \\
& =p \frac{\ell}{k} \frac{(c+\ell+1)^{\gamma}}{k+1}+\left(1-p \frac{\ell}{k}\right) \frac{(c+\ell)^{\gamma}}{k+1}-\frac{(c+\ell)^{\gamma}}{k} \\
& =\frac{(c+\ell)^{\gamma}}{k(k+1)}\left[\ell p\left((1+1 /(c+\ell))^{\gamma}-1\right)-1\right] .
\end{aligned}
$$


Since $(1+y)^{\gamma}-1 \sim \gamma y$ when $y \rightarrow 0+$ and $p \gamma<1$, we can now choose $c$ sufficiently large so that

$$
\ell p\left((1+1 /(c+\ell))^{\gamma}-1\right)<1 \quad \text { for all } \ell \geq 1 ;
$$

then the conditional expectation above is non-positive, and the supermartingale property is established.

Corollary 3.7. Suppose that there exists $c>0$ and $1 \leq \gamma<1 / p$ such that $|F(\omega)| \leq c \omega(1)^{\gamma}$ for every counting function $\omega:[0,1] \rightarrow \mathbb{N}$. Then the convergence stated in Proposition 3.3 holds in $L^{1}(\mathbb{P})$.

Proof. Lemma 3.6 ensures that for any $\gamma^{\prime} \in(\gamma, 1 / p)$,

$$
\sup _{n \geq 1} \mathbb{E}\left[\frac{1}{n} \sum_{j=1}^{n} N_{j}(n)^{\gamma^{\prime}}\right]<\infty
$$

Our statement then follows from a standard argument of uniform integrability. More precisely, we may assume without loss of generality that $F \geq 0$, and then set $F_{b}(\omega)=F(\omega) \wedge b$ for an arbitrary large $b>0$. Next we write

$$
F(\omega)=F_{b}(\omega)+(F(\omega)-b)^{+}
$$

and note that since $F(Y) \leq c Y(1)^{\gamma} \in L^{1}(\mathbb{P})$,

$$
\lim _{b \rightarrow \infty} \mathbb{E}\left[F_{b}(Y)\right]=\mathbb{E}[F(Y)]<\infty .
$$

On the other hand, from our assumptions and Markov's inequality, we have

$$
\mathbb{E}\left[\left(F\left(N_{j}(\lfloor\cdot n\rfloor)\right)-b\right)^{+}\right] \leq c b^{\gamma-\gamma^{\prime}} \mathbb{E}\left[N_{j}(n)^{\gamma^{\prime}}\right],
$$

and it follows from (15) that

$$
\lim _{b \rightarrow \infty} \sup _{n \geq 1} \mathbb{E}\left[\frac{1}{n} \sum_{j=1}^{n}\left(F\left(N_{j}(\lfloor\cdot n\rfloor)\right)-b\right)^{+}\right]=0 .
$$

Since Proposition 3.3 holds for $F_{b}$, this easily entails our claim.

We now have all the ingredients needed for establishing Theorem 3.1

Proof. For every $n \geq 1$, we use the notation with an exponent $(n)$ for quantities related to the skeleton random walk $S^{(n)}$. In particular, $X_{j}^{(n)}=\xi(j / n)-\xi((j-1) / n)$ denotes its $j$ th step, and $N_{j}^{(n)}$ refers to the $j$ th counting process arising in the step reinforcement for $S^{(n)}$.

Fix $k \geq 1, \theta_{1}, \ldots, \theta_{k} \in \mathbb{R}^{d}$ and $t_{1}, \ldots, t_{k} \in[0,1]$, and recall from (14) that

$$
\theta_{j} \cdot \hat{S}^{(n)}\left(\left\lfloor n t_{j}\right\rfloor\right)=\sum_{\ell=1}^{n} N_{\ell}^{(n)}\left(\left\lfloor n t_{j}\right\rfloor\right) \theta_{j} \cdot X_{\ell}^{(n)} .
$$

Recalling from (13) that $\Psi$ denotes the characteristic exponent of $\xi$, we now see by computing first the expectation with respect to the i.i.d. steps that

$$
\mathbb{E}\left[\exp \left\{\mathrm{i} \sum_{j=1}^{k} \theta_{j} \cdot \hat{S}^{(n)}\left(\left\lfloor n t_{j}\right\rfloor\right)\right\}\right]=\mathbb{E}\left[\exp \left\{-\frac{1}{n} \sum_{\ell=1}^{n} \Psi\left(\sum_{j=1}^{k} N_{\ell}^{(n)}\left(\left\lfloor n t_{j}\right\rfloor\right) \theta_{j}\right)\right\}\right] .
$$

This yields us to considering the functional

$$
F(\omega)=\Psi\left(\sum_{j=1}^{k} \omega\left(\left\lfloor n t_{j}\right\rfloor\right) \theta_{j}\right)
$$


where $\omega:[0,1] \rightarrow \mathbb{N}$ stands for a generic counting function. Recall from Lemma 3.1 in [4] that for every $\eta>0$, one has as $|z| \rightarrow \infty$

$$
|\Psi(z)|= \begin{cases}o\left(|z|^{2+\eta}\right) & \text { when } \mathbf{q} \neq 0 \\ o\left(|z|^{\beta(\Lambda)+\eta}\right) & \text { when } \mathbf{q}=0 \text { and } \int_{|x| \leq 1}|x| \Lambda(\mathrm{d} x)=\infty \\ o\left(|z|^{1+\eta}\right) & \text { when } \mathbf{q}=0 \text { and } \int_{|x| \leq 1}|x| \Lambda(\mathrm{d} x)<\infty\end{cases}
$$

Further $\Psi(0)=0$, and observing that

$$
\left|\sum_{j=1}^{k} \omega\left(t_{j}\right) \theta_{j}\right| \leq \omega(1) \sum_{j=1}^{k}\left|\theta_{j}\right|,
$$

we see that the assumption that the memory parameter $p$ is admissible ensures that the requirement of Corollary 3.7 is fulfilled with any $\gamma \in(1,1 / p)$. We conclude that

$$
\lim _{n \rightarrow \infty} \mathbb{E}\left[\exp \left\{\mathrm{i} \sum_{j=1}^{k} \theta_{j} \cdot \hat{S}^{(n)}\left(\left\lfloor n t_{j}\right\rfloor\right)\right\}\right]=\exp \left\{-(1-p) \mathbb{E}\left[\Psi\left(\sum_{j=1}^{k} Y\left(t_{j}\right) \theta_{j}\right)\right]\right\} .
$$

Comparing with Corollary 2.11, this completes the proof.

We now conclude this work by briefly discussing the situation when the memory parameter is not admissible for $(\mathbf{q}, a, \Lambda)$. In this direction, recall that Blumenthal and Getoor [4] also introduced a so-called lower index of a Lévy process $\xi$ with characteristic exponent $\Psi$ as

$$
\beta^{\prime \prime}=\sup \left\{\alpha \geq 0: \lim _{|\theta| \rightarrow \infty}|\theta|^{-\alpha} \Re \Psi(\theta)=\infty\right\} .
$$

There is always the inequality $\beta^{\prime \prime} \leq \beta$, and equality may hold (for instance in the stable case). Now if we assume that $p>1 / \beta^{\prime \prime}$, then we have $\mathbb{E}[\Re \Psi(Y(1))]=\infty$, and it follows from Proposition 3.3 and a classical truncation argument that for every $\theta \in \mathbb{R}^{d} \backslash\{0\}$

$$
\lim _{n \rightarrow \infty} \frac{1}{n} \sum_{j=1}^{n} \Re \Psi\left(N_{j}(n) \theta\right)=\infty, \quad \text { in probability. }
$$

The calculation in the proof of Theorem 3.1 now shows that

$$
\lim _{n \rightarrow \infty} \mathbb{E}\left[\exp \left\{\mathrm{i} \theta \cdot \hat{S}^{(n)}(n)\right\}\right]=0
$$

and hence the sequence of variables $\hat{S}^{(n)}(n)$ cannot converge weakly.

When $\xi$ is an isotropic $\alpha$-stable Lévy process for some $\alpha \in(0,2]$, the upper and lower Blumenthal-Getoor indices are simply $\beta=\beta^{\prime \prime}=\alpha$. Assume that $\alpha>1$ and take any $p>1 / \alpha$. Theorem 1 of Businger [5] states that for every $t \geq 0$, $n^{-p} \hat{S}(\lfloor t n\rfloor)$ converges in probability towards $t^{p} \eta$ as $n \rightarrow \infty$, where $\hat{S}=\hat{S}^{(1)}$ and $\eta$ is some non-degenerate random vector in $\mathbb{R}^{d}$. Since by the scaling property, the processes $\left(\hat{S}^{(n)}(\lfloor t n\rfloor)\right)_{t \geq 0}$ and $\left(n^{-1 / \alpha} \hat{S}(\lfloor t n\rfloor)\right)_{t \geq 0}$ have the same distribution, her result translated in our framework shows that the process $\left(n^{-p+1 / \alpha} \hat{S}^{(n)}(\lfloor t n\rfloor)\right)_{t \geq 0}$ converges in distribution to $\left(t^{p} \eta\right)_{t \geq 0}$ as $n \rightarrow \infty$. A perusal of the arguments of Businger shows that this feature remains provided that $\Psi(c \theta) \sim c^{\alpha} \Psi_{\alpha}(\theta)$ as $c \rightarrow \infty$, where $\Psi_{\alpha}$ is the characteristic exponent of an $\alpha$-stable Lévy process. Nonetheless, it is also seen from her approach that one cannot expect a similar result to hold when $\xi$ is an arbitrary Lévy process, even if we require its upper and lower Blumenthal-Getoor indices $\beta$ and $\beta^{\prime \prime}$ to coincide with $\alpha$.

\section{Acknowledgements}

I would like to thank two anonymous referees for their constructive comments in order to improve the readability of this work. 


\section{References}

[1] O. Angel, N. Crawford and G. Kozma. Localization for linearly edge reinforced random walks. Duke Math. J. 163 (5) (2014) 889-921. MR3189433 https://doi.org/10.1215/00127094-2644357

[2] E. Baur and J. Bertoin. Elephant random walks and their connection to Pólya-type urns. Phys. Rev. E 94 (2016) 052134.

[3] B. Bercu. A martingale approach for the elephant random walk. J. Phys. A 51 (1) (2018) 015201, 16. MR3741953 https://doi.org/10.1088/ 1751-8121/aa95a6

[4] R. M. Blumenthal and R. K. Getoor. Sample functions of stochastic processes with stationary independent increments. J. Math. Mech. 10 (1961) 493-516. MR0123362

[5] S. Businger. The shark random swim (Lévy flight with memory). J. Stat. Phys. 172 (3) (2018) 701-717. MR3827299 https://doi.org/10.1007/ s10955-018-2062-5

[6] S. Businger. The shark random swim (Lévy flight with memory) [v3], 2018. Available at https://arxiv.org/abs/1710.05671. MR3827299 https://doi.org/10.1007/s10955-018-2062-5

[7] C. F. Coletti, R. Gava and G. M. Schütz. Central limit theorem and related results for the elephant random walk. J. Math. Phys. 58 (5) (2017) 053303, 8. MR3652225 https://doi.org/10.1063/1.4983566

[8] C. F. Coletti, R. Gava and G. M. Schütz. A strong invariance principle for the elephant random walk. J. Stat. Mech. Theory Exp. 12 (2017) 123207, 8. MR3748931 https://doi.org/10.1088/1742-5468/aa9680

[9] C. Cotar and D. Thacker. Edge- and vertex-reinforced random walks with super-linear reinforcement on infinite graphs. Ann. Probab. 45 (4) (2017) 2655-2706. MR3693972 https://doi.org/10.1214/16-AOP1122

[10] M. Disertori, C. Sabot and P. Tarrès. Transience of edge-reinforced random walk. Comm. Math. Phys. 339 (1) (2015) 121-148. MR3366053 https://doi.org/10.1007/s00220-015-2392-y

[11] P. Embrechts, C. Klüppelberg and T. Mikosch. Modelling Extremal Events: For Insurance and Finance. Applications of Mathematics (New York) 33. Springer-Verlag, Berlin, 1997. MR1458613 https://doi.org/10.1007/978-3-642-33483-2

[12] S. N. Ethier and T. G. Kurtz. Markov Processes: Characterization and Convergence. Wiley Series in Probability and Mathematical Statistics: Probability and Mathematical Statistics. John Wiley \& Sons, Inc., New York, 1986. MR0838085 https://doi.org/10.1002/9780470316658

[13] O. Kallenberg. Foundations of Modern Probability, 2nd edition. Probability and Its Applications (New York). Springer-Verlag, New York, 2002. MR1876169 https://doi.org/10.1007/978-1-4757-4015-8

[14] R. Kürsten. Random recursive trees and the elephant random walk. Phys. Rev. E 93 (3) (2016) 032111, 11. MR3652690 https://doi.org/10.1103/ physreve.93.032111

[15] C. Mailler and G. Uribe Bravo. Random walks with preferential relocations and fading memory: A study through random recursive trees. J. Stat. Mech. Theory Exp. (2019).

[16] M. Manstavičius and A. Schnurr. Criteria for the finiteness of the strong p-variation for Lévy-type processes. Stoch. Anal. Appl. 35 (5) (2017) 873-899. MR3686473 https://doi.org/10.1080/07362994.2017.1333007

[17] R. Pemantle. A survey of random processes with reinforcement. Probab. Surv. 4 (2007) 1-79. MR2282181 https://doi.org/10.1214/07-PS094

[18] C. Sabot and P. Tarrès. Edge-reinforced random walk, vertex-reinforced jump process and the supersymmetric hyperbolic sigma model. J. Eur. Math. Soc. (JEMS) 17 (9) (2015) 2353-2378. MR3420510 https://doi.org/10.4171/JEMS/559

[19] G. M. Schütz and S. Trimper. Elephants can always remember: Exact long-range memory effects in a non-Markovian random walk. Phys. Rev. E 70 (2004) 045101.

[20] H. A. Simon. On a class of skew distribution functions. Biometrika 42 (3/4) (1955) 425-440. MR0073085 https://doi.org/10.1093/biomet/42.3-4. 425 